\title{
What does entropy reveal in phase analysis of myocardial perfusion SPECT?
}

\author{
Koichi Okuda, PhD, ${ }^{a}$ and Kenichi Nakajima, $M D, \mathrm{PhD}^{\mathrm{b}}$ \\ a Department of Physics, Kanazawa Medical University, Kahoku, Ishikawa, Japan \\ b Department of Functional Imaging and Artificial Intelligence, Kanazawa University, Kanazawa, \\ Japan
}

Received Jun 17, 2019; accepted Jun 17, 2019

doi: $10.1007 / \mathrm{s} 12350-019-01813-w$

\section{See related article, pp. 162-171}

Rudolf Clausius described entropy for thermodynamics in 1865, and Ludwig Boltzmann, Josiah Willard Gibbs, James Clerk Maxwell, and others expanded the concept into statistical mechanics. Claude Elwood Shannon also introduced a new aspect of entropy into information theory during $1948 .^{1}$ Consequently, the long, notable history of entropy studies has facilitated understanding of the laws of nature. Among several equations for entropy that are applied across many scientific disciplines, the Shannon equation $\left(H=-\sum p_{i}\right.$ $\log p_{i}$, where $p_{i}$ is the probability of state $i$ ) that is based on information theory is applied to phase analyses of gated single-photon emission computed tomography (SPECT). In phase histograms generated from gated SPECT data, $p_{i}$ represents the frequency of the histogram bin $i$. Entropy increases if the phase distribution becomes disordered in histograms. If the equation for entropy is divided by $\log (n)$, in which $n$ represents the number of histogram bins, the entropy range is $0-1$, which represents total order to disorder.

A study by Shimizu and colleagues published in this issue of the Journal of Nuclear Cardiology showed that entropy and estimated glomerular filtration rates (eGFR) could predict a poor cardiac prognosis in patients with complete left bundle branch block (CLBBB). ${ }^{2}$ High entropy and low eGFR were independent predictors determined by Cox regression and Kaplan-Meyer

Reprint requests: Koichi Okuda, PhD, Department of Physics, Kanazawa Medical University, 1-1 Daigaku, Uchinada, Kahoku, Ishikawa 920-0293, Japan; okuda@nmd.m.kanazawa-u.ac.jp

J Nucl Cardiol 2021;28:172-4.

1071-3581/\$34.00

Copyright (C) 2019 American Society of Nuclear Cardiology. analyses for major cardiac events (MACE) in these patients. Moreover, Random Forest machine learning also clarified entropy and eGFR as independent predictors of MACE. Others have used left ventricular (LV) volumes, LV ejection fraction, echocardiographic parameters, and clinical demographics as predictors of heart disease. Although entropy might be able to clarify the pathophysiology of patients with CLBBB, whether it implies causative indices and provides clinical information that is directly related to pathophysiology cannot be determined. This is because physical aspects of acquisition and processing along with patient-associated factors can influence entropy, which is also susceptible to LV volume, LV ejection fraction, age, statistical noise, and heart failure. ${ }^{3}$

Since the phase distribution derived from gated SPECT images can easily be influenced by acquisition conditions and patient characteristics, entropy includes many kinds of artifacts in addition to bandwidth and phase standard deviation (SD). Previous studies have shown that the amount of time required to acquire SPECT images (acquisition time) considerably influences phase analysis. Decreasing acquisition time is associated with increased entropy in clinical patients. ${ }^{4}$ Reducing the amount of acquisition time by $50 \%$ changed mean entropy from $55.0 \% \pm 6.41 \%$ to $59.5 \% \pm 6.06 \%(p<0.002)$. Moreover, decreased gated SPECT counts were also associated with increased entropy in a phantom study. ${ }^{5} \mathrm{~A}$ decrease in the average SPECT count per pixel from 97.7 to 17.8 increased entropy from 0.26 to 0.35 . Acquisition orbits of $180^{\circ}$ and $360^{\circ}$ significantly influenced bandwidth and phase SD in a clinical study. ${ }^{6}$ Notably, sex influences phase parameters. ${ }^{7}$ When male and female LV end-diastolic volumes were matched in a normal database, entropy was significantly higher in males than in females $(26.3 \% \pm 8.0 \%$ vs $20.1 \% \pm 5.5 \%, p=0.020)$. Moreover, the amount of injected radionuclide, stress or rest 

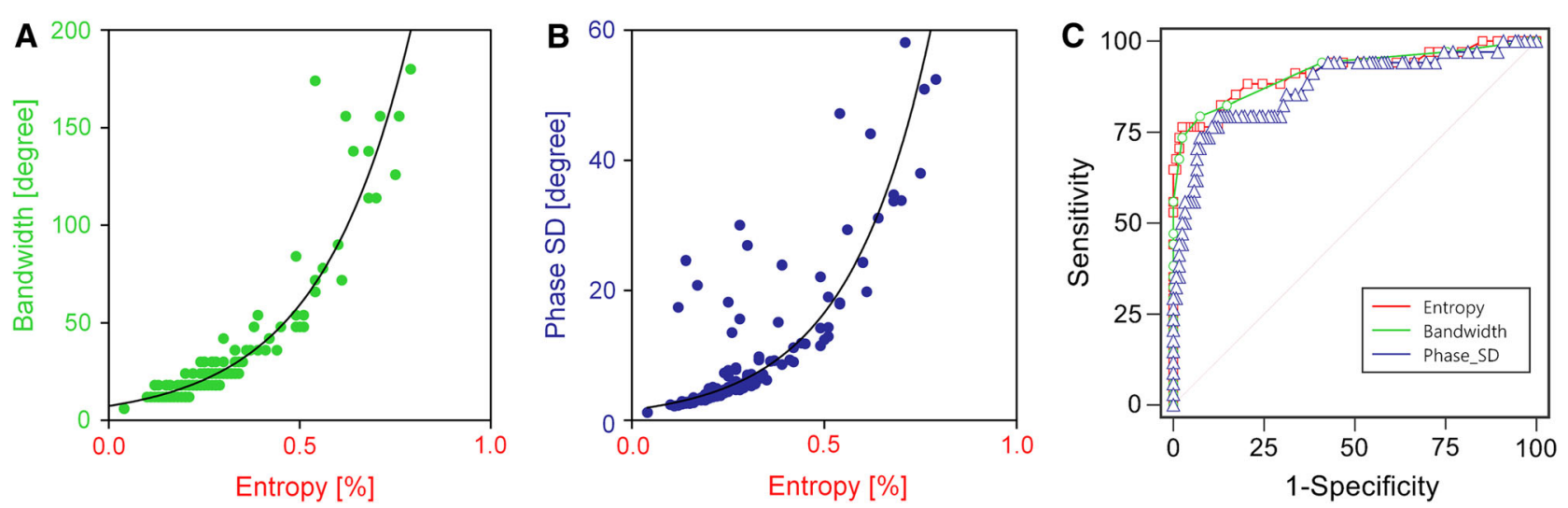

Figure 1. Relationships among phase SD, entropy, and bandwidth. Relationships are exponential between entropy and (A) bandwidth $\left(Y=5.5+0.025 * X^{2}, R^{2}=0.87\right)$ and (B) phase SD $\left(Y=1.25+0.0075 * X^{2}, R^{2}=0.75\right)$. Areas under receiver operating characteristics curves $(\mathbf{C})$ are smaller for phase SD $(0.88 \pm 0.39)$ than for entropy $(0.92 \pm 0.034, p=0.016)$ and bandwidth $(0.92 \pm 0.033, p=0.0047)$. Data are shown as means \pm standard error. $S D$, standard deviation.

acquisition, the number of frames per cardiac cycle, and the type of software used for phase analysis ${ }^{7,8}$ are important when computing entropy.

To understand relationships among bandwidth, phase $\mathrm{SD}$, and entropy, the following example is extracted from our published work. ${ }^{8}$ Among 156 patients who were assessed by ${ }^{99 \mathrm{~m}} \mathrm{Tc}$-sestamibi or ${ }^{99 \mathrm{~m}}$ Tc-tetrofosmin gated SPECT imaging, 122 were diagnosed with normal perfusion and cardiac function, and 34 patients with anomalous cardiac function had suspected LV dyssynchrony. Entropy was exponentially associated with bandwidth (Figure 1a) and phase SD (Figure $1 b$ ). Figure $1 \mathrm{~b}$ shows some outliers of phase SD in the lower range (0.0-0.5) of entropy, whereas Figure 1a does not. Due to being calculated using all histogram bins, phase SD detects all noises or outliers, and thus increases. Consequently, in a comparison of the ability of three receiver operating characteristics (ROC) curves to detect LV dyssynchrony, the area under the ROC curves was smaller for phase SD than for entropy and bandwidth (Figure 1c). These results showed that entropy and bandwidth were superior to phase SD for phase analysis.

Left ventricular dyssynchrony has been investigated in patients with $\mathrm{CLBBB}^{2}$ and in those with chronic kidney disease and normal perfusion defect scores. ${ }^{9}$ Both studies used gated SPECT imaging and phase analysis and found entropy, bandwidth, and phase SD useful. Whether disordered contraction timing within the myocardium has pathophysiological significance that reflects underlying myocardial damage is of interest. Entropy might be a promising parameter of prognosis; however, whether it contains truly causative factors that are indeed associated with prognosis or simply reflects composite factors derived from the heterogeneous distribution of myocardial uptake remains unknown. Although mechanical dyssynchrony has been evaluated with the bandwidth and phase SD of phase histograms in most studies, entropy might yield new aspects of phase analysis. However, entropy is so enigmatic that its value should be carefully considered. A phase analysis study of entropy in myocardial perfusion SPECT would be worthy of consideration, and the true role of entropy will only be confirmed by accumulated diagnostic and prognostic clinical experiences.

\section{Acknowledgments}

This study was partly funded by the JSPS KAKENHI Grants (Number 18K15649). The authors appreciate the editorial assistance of Norma Foster.

\section{Disclosures}

The authors have no conflicts of interest to declare.

\section{References}

1. Shannon CE. A mathematical theory of communication. Bell Syst Tech J. 1948;27:379-423.

2. Shimizu M, Iiya M, Fujii H, Kimura S, Suzuki M, Nishizaki M. Left ventricular end-systolic contractile entropy can predict cardiac prognosis in patients with complete left bundle branch block. J Nucl Cardiol 2019.

3. Nakajima K, Okuda K, Matsuo S, Slomka P. Making the invisible visible: Phase dyssynchrony has potential as a new prognostic marker. J Nucl Cardiol. 2019;26:298-302.

4. Kortelainen MJ, Koivumaki TM, Vauhkonen MJ, Hakulinen MA. Dependence of left ventricular functional parameters on image 
acquisition time in cardiac-gated myocardial perfusion SPECT. J Nucl Cardiol. 2015;22:643-51.

5. Kita A, Onoguchi M, Shibutani T, Sugimoto K, Kosaka N, Adachi $\mathrm{T}$, et al. Influence of myocardial count on phase dyssynchrony analysis of gated myocardial perfusion single-photon emission computed tomography. Nucl Med Commun. 2019;40:124-30.

6. Misaka T, Hosono M, Kudo T, Ito T, Syomura T, Uemura M, et al. Influence of acquisition orbit on phase analysis of gated single photon emission computed tomography myocardial perfusion imaging for assessment of left ventricular mechanical dyssynchrony. Ann Nucl Med. 2017;31:235-44.

7. Nakajima K, Okuda K, Matsuo S, Kiso K, Kinuya S, Garcia EV. Comparison of phase dyssynchrony analysis using gated myocardial perfusion imaging with four software programs: Based on the
Japanese Society of Nuclear Medicine working group normal database. J Nucl Cardiol. 2017;24:611-21.

8. Okuda K, Nakajima K, Matsuo S, Kashiwaya S, Yoneyama H, Shibutani T, et al. Comparison of diagnostic performance of four software packages for phase dyssynchrony analysis in gated myocardial perfusion SPECT. EJNMMI Res. 2017;7:27.

9. Mori H, Isobe S, Suzuki S, Unno K, Morimoto R, Kano N, et al. Prognostic value of left ventricular dyssynchrony evaluated by gated myocardial perfusion imaging in patients with chronic kidney disease and normal perfusion defect scores. J Nucl Cardiol. 2019;26:288-97.

Publisher's Note Springer Nature remains neutral with regard to jurisdictional claims in published maps and institutional affiliations. 